\title{
AVALIAÇÃO DA INTERAÇÃO PARTÍCULA-AGLOMERANTE NO PROCESSO DE PELOTIZAÇÃO DE MINÉRIO DE FERRO*
}

\author{
Sandra Lúcia de Moraes ${ }^{1}$ \\ José Renato Baptista de Lima² \\ João Batista Ferreira Neto ${ }^{3}$ \\ Cátia Fredericci ${ }^{4}$ \\ Eduardo Maziero Saccoccio 5
}

\section{Resumo}

Vários trabalhos voltados a resolução dos problemas da utilização de bentonita no processo de aglomeração de minério de ferro referem-se ao emprego de aglomerantes orgânicos, sendo que os dois tipos principais são os à base de celulose e de poliacrilamidas. Este trabalho teve como objetivo um melhor entendimento da ação de um aglomerante à base de carboximetilcelulose no ganho de resistência mecânica da pelota. Foi avaliada a interação entre a superfície mineral de dois concentrados tipo pellet feed brasileiros e os componentes presentes no aglomerante Peridur de uso comercial. $O$ método proposto e desenvolvido no presente trabalho permitiu comprovar que um mesmo aglomerante atua de maneira diferenciada dependendo do tipo de minério de ferro empregado. Isto reforça a importância do entendimento de como cada produto, utilizado como aglomerante no processo de pelotização, age nos constituintes do minério de ferro para promover o incremento necessário de resistência mecânica das pelotas.

Palavras-chave: Pelotização; Minério de ferro; Aglomerante; Interação.

\section{EVALUATION OF INTERACTION PARTICLE-BINDER IN THE IRON ORE PELLETIZING PROCESS}

\section{Abstract}

The usage of organic binders in iron ore agglomeration is related by several studies, which aim was to solve the problems of using bentonite in such process. The two main types of organic binders are derived from cellulose and polyacrylamide. The goal of this work was to improve the understanding of how a carboxymethyl cellulose-based organic binder acts to enhance the mechanical properties of iron ore pellets, by assessing the interaction between the mineral surface of two Brazilian iron ore concentrates (pellet feed) and the components present in the commercial binder Peridur. The method proposed and developed in this study showed that the same binder acts differently depending on the type of iron ore used. This result reinforces the importance of understanding how each product used as binder in the pelletizing process acts in the iron ore constituents to promote the necessary increase in mechanical strength of the pellets.

Keywords: Pelletizing; Iron ore; Binder; Interaction.

1 Engenheira Química, Doutora, Pesquisadora, Centro de Tecnologia em Metalurgia e Materiais, Instituto de Pesquisas Tecnológicas do Estado de São Paulo, São Paulo, SP, Brasil.

2 Engenheiro de Minas, Professor Doutor, Departamento de Engenharia de Minas e de Petróleo, Escola Politécnica da Universidade de São Paulo, SP, Brasil.

3 Engenheiro Metalurgista, Doutor, Pesquisador, Centro de Tecnologia em Metalurgia e Materiais, Instituto de Pesquisas Tecnológicas do Estado de São Paulo, São Paulo, SP, Brasil.

4 Química, Doutora, Pesquisadora, Centro de Tecnologia em Metalurgia e Materiais, Instituto de Pesquisas Tecnológicas do Estado de São Paulo, São Paulo, SP, Brasil.

5 Químico ambiental, Pesquisador Assistente, Centro de Tecnologia em Metalurgia e Materiais, Instituto de Pesquisas Tecnológicas do Estado de São Paulo, São Paulo, SP, Brasil. 


\section{INTRODUÇÃO}

Estudos visando a substituição da bentonita como aglomerante no processo de aglomeração de minério de ferro vêm sendo realizados desde a década de 70 . As vantagens apresentadas pelos aglomerantes orgânicos, tais como não inserirem contaminantes durante o processo, serem utilizados em menor quantidade e de poderem ser eliminados de forma substancial durante a queima das pelotas, levaram a uma série de pesquisas de novos produtos para este fim, conforme reportado por Moraes [1].

Observa-se em muitas patentes e artigos científicos sobre o tema [1], que grande parte dos desenvolvimentos é realizada com certo grau de empirismo, ou seja, não se constata uma fundamentação teórica da ação destes materiais nos mecanismos de formação e, consequentemente, sua influência nas propriedades mecânicas das pelotas produzidas.

Conforme já abordado por Mourão [2], a gênese do minério tem influência no teor ótimo de umidade e influi nos níveis de adição de aglomerantes, consequentemente influi nos indicadores de qualidade das pelotas "verdes". Este fato reforça a importância de se ter conhecimento de como estes materiais interagem com os diferentes tipos de minério de ferro que vêm sendo processados. Neste contexto, o estudo de interações entre a superfície mineral e os componentes presentes nos aglomerantes pode ajudar no entendimento do processo de aglomeração para cada tipo minério de ferro.

A adsorção é um processo de interação de uma espécie química entre duas fases que permite a redução da energia livre superficial [3]. No caso das superfícies minerais, as interações de interesse entre surfactantes e polímeros se dão na interface líquido/sólido. A adsorção pode ser física ou química e o processo é governado por um grande número de interações na região interfacial, envolvendo muitas variáveis tais como: solubilidade e carga superficial do sólido, $\mathrm{pH}$, distribuição do tamanho de partículas e a própria estrutura da espécie química que se adsorve [3].

Conceitualmente, a exposição de um sólido a um líquido polar (por exemplo, a água) possibilita à superfície do sólido adquirir uma carga superficial, a qual pode ser originada da ionização dos átomos da superfície, da adsorção de íons da solução ou a dissolução de íons que compõem a superfície [4]. Sendo assim, dependendo das forças de ligação que contribuem para a adsorção ela poderá ocorrer de duas maneiras: química ou física. Adsorção química ocorre quando um adsorbato forma uma ligação covalente com a superfície do adsorvente enquanto que a adsorção física implica na contribuição de forças mais fracas, tais como interações de van der Waals.

Neste caso, uma ferramenta que pode ajudar a entender essas interações é a espectroscopia de infravermelho por transformada de Fourier (FTIV). A FTIV se baseia na capacidade das moléculas em sofrer transições de um estado de energia vibracional para outro, absorvendo a radiação infravermelha. A espectroscopia vibracional mede diferentes tipos de vibração entre átomos de acordo com suas ligações interatômicas. Diferentes ligações químicas possuem diferentes frequências vibracionais. O espectro no infravermelho indica a presença destes diferentes tipos de ligações nas moléculas. Portanto, cada molécula absorve a radiação em uma determinada frequência dependendo da sua natureza. Essa absorção de radiação resulta em um número de bandas de absorção localizadas em diferentes frequências do espectro [5]. Supondo que os grupos funcionais de duas substâncias interajam, 
pode ocorrer mudança perceptível na localização de sua banda de absorção em relação às substâncias isoladas.

Neste trabalho são apresentados os resultados do estudo que visou o melhor entendimento dos mecanismos da ação de aglomerantes orgânicos na aglomeração de pelotas de concentrado de minério de ferro por meio da avaliação da sua influência na interação partícula-aglomerante.

\section{MATERIAIS E MÉTODOS}

Foram estudados dois concentrados de minério de ferro brasileiros tipo pellet feed, oriundos da indústria, denominados Minério A e Minério B, cujas distribuições granulométricas são apresentadas na Figura 1. Como se pode observar na Figura 1, o Minério A é mais fino que o Minério $B$, com $P_{80}$ de 43,8 $\mu \mathrm{m}$, enquanto o $P_{80}$ do Minério A é $54,4 \mu \mathrm{m}$.

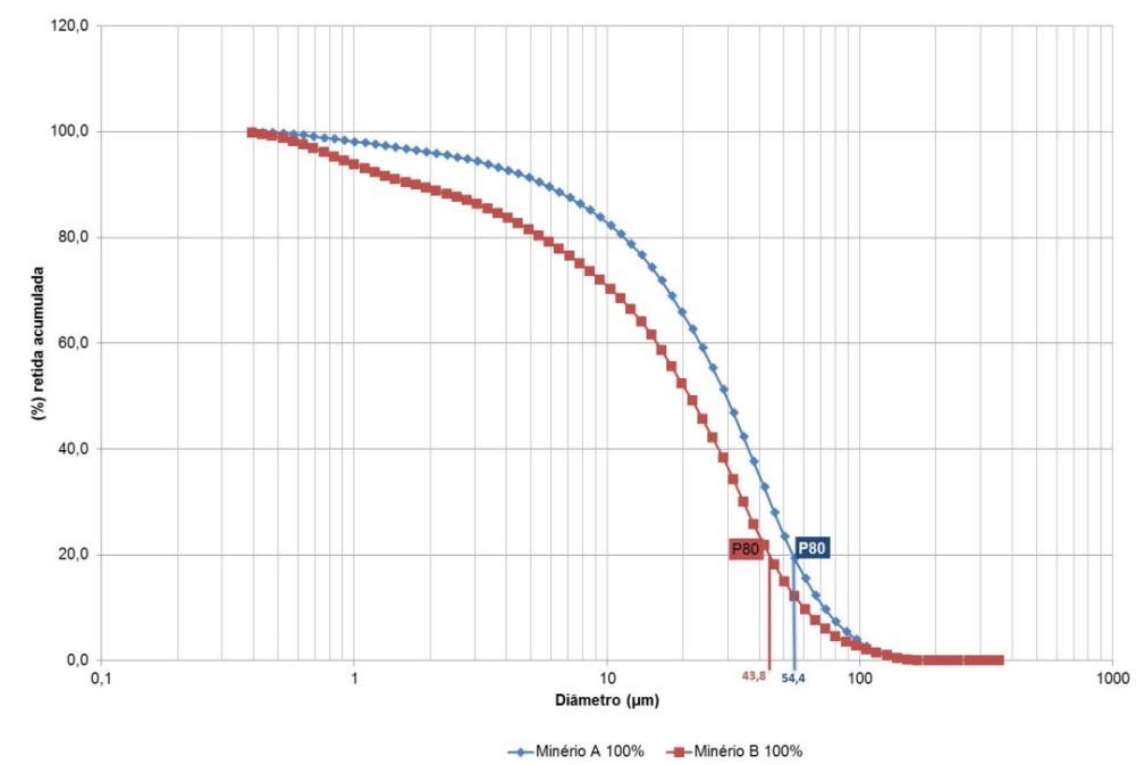

Figura 1. Análise granulométrica dos concentrados de minério de ferro estudados.

A Figura 2 apresenta o difratograma dos minérios de ferro A e B obtidos em equipamento Shimadzu, modelo XRD 6000, com radiação Ka do Cu e varredura de $1 \%$ min. As fichas do Joint Committee for Powder Diffraction Standards (JCPDS) utilizadas para a identificação das fases foram: 81-0464 $(\mathrm{FeO}(\mathrm{OH}))$, 77-1545 $\left(\mathrm{Fe}_{3} \mathrm{O}_{4}\right)$ e 87-1165 $\left(\mathrm{Fe}_{2} \mathrm{O}_{3}\right)$.

Como é possível observar na Figura 2, a distribuição dos picos nos difratogramas dos minérios de ferro $\mathrm{A}$ e $\mathrm{B}$ aponta a presença de três diferentes tipos de óxidos de ferro na sua composição, quais sejam: hematita $\left(\mathrm{Fe}_{2} \mathrm{O}_{3}\right)$, goethita $(\mathrm{FeO}(\mathrm{OH}))$ e magnetita $\left(\mathrm{Fe}_{3} \mathrm{O}_{4}\right)$. Porém, os picos relacionados à goethita são mais intensos no difratograma do minério $B$ em comparação ao difratograma do minério $A$, podendo indicar que a goethita esteja presente em maior quantidade no minério $B$.

O aglomerante estudado foi o orgânico de uso comercial Peridurß 330, fabricado pela Akzo Nobel, usado na proporção de $0,04 \%$ em peso sobre a massa de concentrado de minério de ferro. No caso do minério $A$, avaliou-se também a utilização do aglomerante Peridur em conjunto com $\mathrm{NaOH}$, na proporção de $0,02 \%$ em peso sobre a massa de concentrado de minério de ferro, como um possível aditivo do aglomerante. 


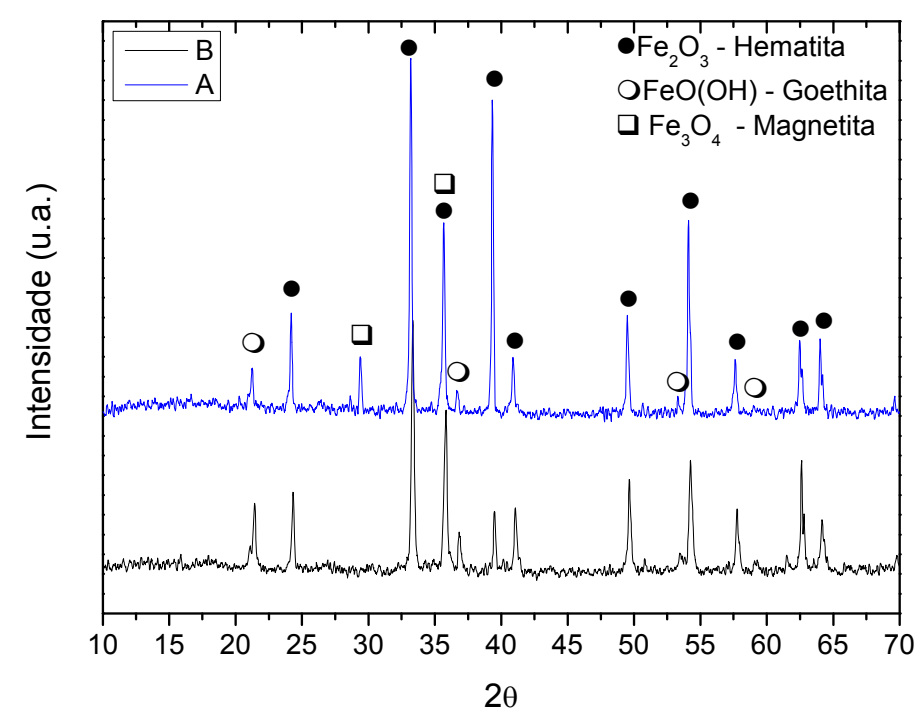

Figura 2. Difratograma de raios- $X$ dos minérios de ferro $A$ e $B$.

A avaliação da interação partícula-aglomerante foi realizada por meio de espectros de infravermelho, obtidos em espectrofotômetro marca Thermo Scientific, modelo Nicolet 6700 FT-IR.

\section{RESULTADOS E DISCUSSÃO}

\subsection{Avaliação das Pelotas Obtidas - Minério A e Minério B Utilizando o Aglomerante Peridur}

Os resultados de resistência à compressão das pelotas confeccionadas com os Minérios A e B utilizando o aglomerante Peridur são apresentados na Tabela 1.

Conforme se observa na Tabela 1 , a adição de $\mathrm{NaOH}$ em conjunto com o aglomerante Peridur promove um incremento de resistência à compressão da pelota verde confeccionada com o Minério A em cerca de $56 \%$, possivelmente pelo fato do $\mathrm{NaOH}$ estar atuando como um aditivo do aglomerante.

Tabela 1 Umidade, resistência a quedas e resistência à compressão das pelotas verde, seca e queimada, confeccionadas com os minérios A e B na presença de aglomerantes nas concentrações indicadas

\begin{tabular}{|c|c|c|c|c|c|}
\hline \multirow[t]{2}{*}{ Ensaio } & \multirow[t]{2}{*}{ Composição } & \multirow{2}{*}{$\begin{array}{c}\text { Umidade } \\
(\%)\end{array}$} & \multirow{2}{*}{$\begin{array}{c}\text { Quedas } \\
(45 \mathrm{~cm}) \pm \mathrm{P}_{95}\end{array}$} & \multicolumn{2}{|c|}{$\begin{array}{c}\text { Resistência á compressão } \\
\text { (kgf/pel) } \pm \mathrm{P}_{95}\end{array}$} \\
\hline & & & & verde & seca \\
\hline 3 & Minério A+Peridur $(0,04 \%)$ & 11,47 & $5,4 \pm 0,73$ & $1,01 \pm 0,10$ & $1,59 \pm 0,12$ \\
\hline 9 & $\begin{array}{l}\text { Minério A+Peridur }(0,04 \%)+ \\
\mathrm{NaOH}(0.02 \%)\end{array}$ & 10,95 & $6,3 \pm 1,65$ & $1,58 \pm 0,11$ & $6,57 \pm 0,67$ \\
\hline 13 & Minério B+Peridur $(0,04 \%)$ & 9,93 & $4,7 \pm 0,88$ & $1,78 \pm 0,21$ & $6,86 \pm 0,74$ \\
\hline
\end{tabular}

Embora o aglomerante Peridur tenha proporcionado a estabilidade da pelota verde para os dois minérios estudados, o mesmo não foi capaz de garantir a ganho de resistência à compressão da pelota na etapa de secagem para o concentrado de minério de ferro $\mathrm{A}$. No entanto, o emprego do aglomerante Peridur em conjunto com o $\mathrm{NaOH}$ proporcionou um incremento significativo da resistência à compressão da pelota seca (6,58 $\mathrm{kgf} /$ pelota) para o mesmo Minério A avaliado, a qual é cerca de 1,58 vezes superior ao mínimo estipulado. Com o objetivo de avaliar a interação partícula-aglomerante e a influência do $\mathrm{NaOH}$ na resistência à seco das pelotas, foi 
realizada uma série de estudos de interação analisando os espectros de infravermelho dos concentrados de minério de ferro, do aglomerante e da mistura concentrado de minério ferro e aglomerante.

\subsection{Avaliação da Interação Partícula-Aglomerante}

Os espectros de infravermelho do Minério $A$ e do Minério $B$ são apresentados na Figura 2. Como pode ser observado na Figura 2(a), para o Minério $A$, as bandas de absorção encontradas em 466 e $546 \mathrm{~cm}^{-1}$ correspondem às vibrações ligações $\mathrm{Fe}-$ O. Outra banda em destaque está em $3448 \mathrm{~cm}^{-1}$ e pode estar relacionada às vibrações do grupo $\mathrm{OH}$ da água ou à presença de grupos $\mathrm{OH}$ na superfície [6].

No caso do Minério B, cujos espectros são apresentados na Figura 2(b), foram encontradas bandas de absorção correspondentes à ligação $\mathrm{Fe}-\mathrm{O}$ em 463 e 544 $\mathrm{cm}^{-1}$ [7]. Outras duas bandas de absorção principais localizadas em 895 e $800 \mathrm{~cm}^{-1}$ podem corresponder às vibrações das ligações $\mathrm{Fe}-\mathrm{O}-\mathrm{H}$ na goethita $(\mathrm{FeO}(\mathrm{OH}))$. Além destas, são observadas outras duas bandas de absorção nas regiões de 3440 e $3125 \mathrm{~cm}^{-1}$. A banda de absorção na região de $3440 \mathrm{~cm}^{-1}$ pode ser correspondente às vibrações do grupo $\mathrm{OH}$ da água ou à presença de grupos $\mathrm{OH}$ na superfície e a banda na região de $3125 \mathrm{~cm}^{-1}$ pode ser atribuída às vibrações do grupo $\mathrm{OH}$ na estrutura da goethita [6-8].

Na Figura 4 é apresentado o espectro de infravermelho do aglomerante orgânico utilizado. Cabe ressaltar que o aglomerante orgânico Peridur é composto por uma mistura de carboximetilcelulose $(\mathrm{CMC})$, carbonato de sódio $\left(\mathrm{Na}_{2} \mathrm{CO}_{3}\right)$ e cloreto de sódio $(\mathrm{NaCl})$.

Como é possível observar na Figura 4, as bandas de absorção localizadas em 702 e $881 \mathrm{~cm}^{-1}$ correspondem às vibrações da ligação do carbonato de sódio [9]. As outras bandas de absorção são principalmente correspondentes às ligações da CMC. A banda de absorção em $1159 \mathrm{~cm}^{-1}$ pode ser atribuída às vibrações das ligações $\mathrm{C}-\mathrm{O}$ e dos grupos C-O-C. A absorção na região de $1770 \mathrm{~cm}^{-1}$ refere-se às vibrações do grupo $\mathrm{C}=\mathrm{O}$. Assim como nos minérios, observa-se absorção na região de $3462 \mathrm{~cm}^{-1}$ que podem ser originadas das vibrações do grupo $\mathrm{OH}$ da água. Além disso, pode-se observar nas regiões de 1617 e $1448 \mathrm{~cm}^{-1}$ a absorção das variações simétricas e assimétricas dos grupos $\mathrm{COO}^{-}$, respectivamente [10].

Em relação ao estudo da interação entre os dois tipos de minério de ferro com o aglomerante orgânico, as principais variações observadas foram nas bandas de absorção do grupo COO- simétrico. A Figura apresenta os espectros de infravermelho das amostras de Minério de ferro A com aglomerante orgânico (Figura a), Minério de ferro A com aglomerante orgânico e adição de $\mathrm{NaOH}$ (Figura b) e Minério A com o aglomerante orgânico (Figura c). 


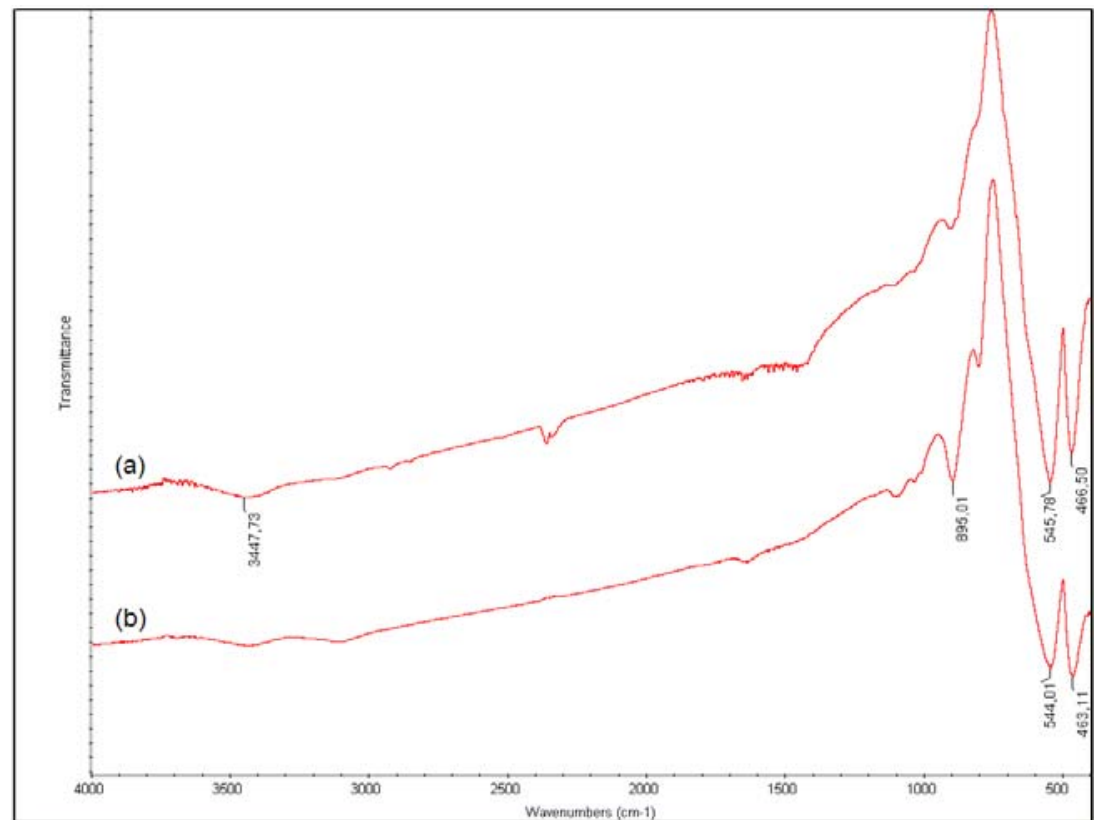

Figura 2. Espectro de infravermelho dos minérios analisados. (a) Minério $A$ e (b) Minério $B$.

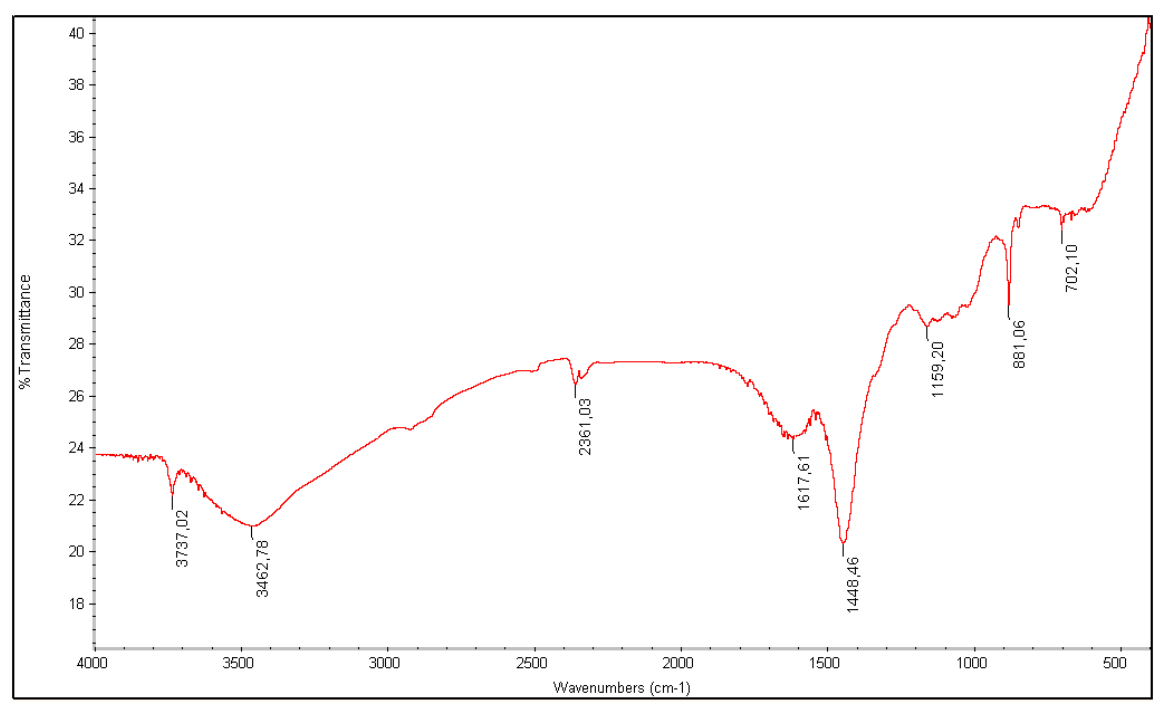

Figura 4. Espectro de infravermelho do aglomerante orgânico Peridur. 


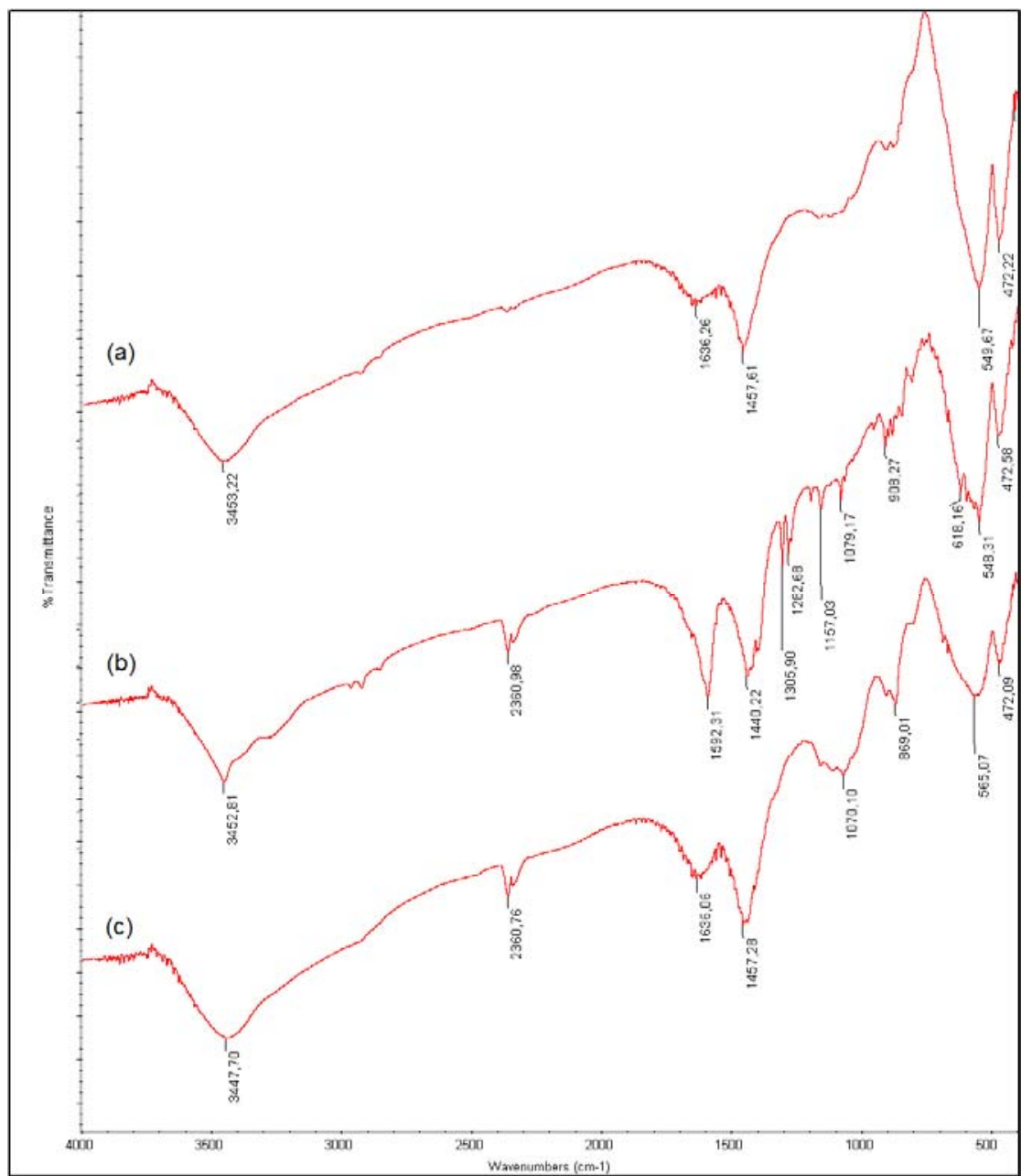

Figura 5. Espectros de infravermelho das misturas de minério com o aglomerante orgânico. (a) Minério A com aglomerante orgânico; (b) Minério A com aglomerante orgânico e adição de $\mathrm{NaOH}$; e (c) Minério B com o aglomerante orgânico.

Os valores das bandas de absorção do grupo COO- foram sumarizados na Tabela e comparados com o valor da banda de absorção do grupo COO- simétrico no aglomerante orgânico Peridur.

Tabela 2. Valores das bandas de absorção do grupo COO- em comparação ao valor da banda de absorção do grupo $\mathrm{COO}^{-}$simétrico no aglomerante orgânico Peridur

\begin{tabular}{|c|c|c|}
\hline Amostra & $\begin{array}{c}\text { Banda de absorção do grupo } \\
\text { COO- } \\
\left(\mathrm{cm}^{-1}\right)\end{array}$ & $\begin{array}{c}\text { Variação em relação a posição } \\
\text { do grupo } \mathrm{COO}^{-} \text {no Peridur® } \\
\left(\mathrm{cm}^{-1}\right)\end{array}$ \\
\hline Peridur® & 1618 & ------- \\
\hline Minério A + Peridur $\circledR$ & 1636 & 19 \\
\hline Minério $A+$ Peridur $®+\mathrm{NaOH}$ & 1592 & -25 \\
\hline Minério B + Peridur® & 1636 & 18 \\
\hline
\end{tabular}

A banda de absorção atribuída ao grupo COO- é deslocada para $1636 \mathrm{~cm}^{-1}$ para as composições de minério de ferro $A$ e $B$ com aglomerante sem adição de $\mathrm{NaOH}$, e para $1592 \mathrm{~cm}^{-1}$ para a composição do Minério A com aglomerante $+\mathrm{NaOH}$. Estas variações na localização das bandas sugerem interações entre a superfície do minério de ferro A e o grupo funcional COO- [11]. Os Minérios de ferro A e B apresentam goethita com ligações $\mathrm{Fe}-\mathrm{O}-\mathrm{H}$. No entanto, como mostra os difratogramas de raios-X de ambos (Figura 2), o Minério B apresenta o pico situado 
em $21,2^{\circ}(2 \theta)$, característico da goethita, em maior intensidade que o mesmo pico no difratograma do Minério A. Esta observação pode ser um indicativo de que o Minério $\mathrm{B}$ apresenta maior quantidade de $\mathrm{FeOOH}$. Se a aglomeração ocorre devido às interações dos grupos ${ }^{-} \mathrm{OH}$ da superfície do minério com o grupo funcional =O-C-Oda carboximetilcelulose, espera-se maior número de interações entre os mesmos, formando ligações de hidrogênio com o grupo funcional $\mathrm{COO}^{-}$, que podem ser representadas como na Figura .

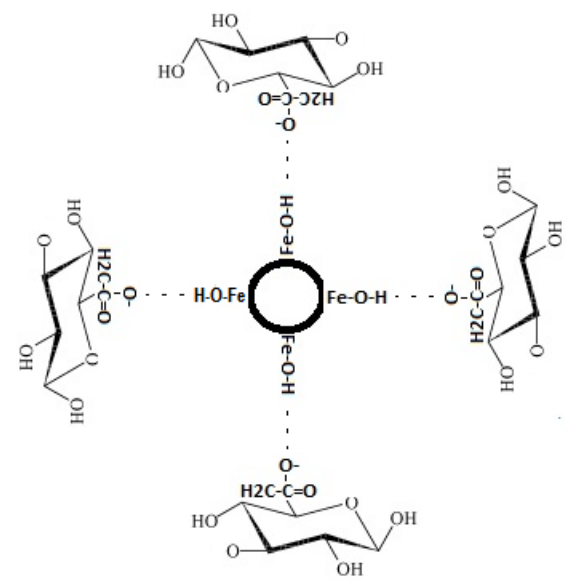

Figura 6. Representação esquemática da interação de moléculas de CMC com a superfície de minério de ferro.

A adição de $\mathrm{NaOH}$ no Minério $A$, durante o processo de aglomeração, pode contribuir para a formação de mais sítios ${ }^{-} \mathrm{OH}$ na superfície deste minério. Com maior número de ligações entre os grupos ${ }^{-} \mathrm{OH}$ do minério e de $\mathrm{COO}^{-}$da carboximetilcelulose, há maior interação entre minério e aglomerante, conferindo maior resistência aos aglomerados, cuja composição é formulada com adição de hidróxido de sódio.

Um fato interessante observado no espectro da mistura de Minério $\mathrm{A}+$ Peridur+ $\mathrm{NaOH}$ (Figura b) é que a banda relacionada ao grupo $\mathrm{COO}^{-}$desloca-se para frequência menor $\left(1.592 \mathrm{~cm}^{-1}\right)$ em relação a mesma do espectro do aglomerante Peridur (1.632 $\mathrm{cm}^{-1}$ ). Esta banda é relacionada ao estiramento assimétrico de $\mathrm{COO}$. Se muitos sítios $\mathrm{OH}$ são formados na superfície e muitas moléculas de $\mathrm{CMC}$ formam ligações com os mesmos, pode ocorrer aproximação entre as moléculas de CMC, dificultando a vibração do estiramento assimétrico, demandando maior energia.

A maior interação entre o Minério $A$ e o aglomerante orgânico se deu pela adição de grupos $\mathrm{OH}$ na estrutura superficial do minério. A adição de grupos $\mathrm{OH}$ possibilitou a formação de ligação entre os grupos $\mathrm{OH}$ na superfície do minério e os grupos $\mathrm{COO}^{-}$ do aglomerante. Atribui-se a essa maior interação os melhores resultados de resistência à compressão.

Já o Minério $B$ possui maior quantidade de grupos $\mathrm{OH}$ em sua estrutura, devido a presença mais acentuada de goethita $(\mathrm{FeO}(\mathrm{OH}))$ em sua composição. A presença natural de grupos $\mathrm{OH}$ na superfície do Minério $\mathrm{B}$ promove uma interação com os grupos COO- do aglomerante orgânico de tal forma que dispensa a utilização de $\mathrm{NaOH}$ na mistura. 


\section{CONCLUSÃO}

Dos trabalhos realizados tem-se que:

- o grau de interação partícula-aglomerante depende das características do minério empregado que, no caso dos minérios estudados, está associada à quantidade de grupos $\mathrm{OH}$ existentes na estrutura do minério. No caso avaliado, a interação partícula-aglomerante possibilitou a formação de pontes de hidrogênio entre os grupos $\mathrm{OH}^{-}$da superfície do minério e os grupos $\mathrm{COO}^{-}$simétricos do aglomerante;

- os diferentes resultados apresentados pelos dois concentrados de minério de ferro avaliados quanto a interação partícula-aglomerante reforçam a necessidade de se entender melhor como cada aglomerante utilizado exerce influência na promoção de ganho de resistência das pelotas durante o processo de pelotização por tipo de minério empregado, especialmente na etapa de processo da pelota crua.

\section{Agradecimentos}

Os autores agradecem a FAPESP - Fundação de Amparo à Pesquisa do Estado de São Paulo pelo suporte financeiro a este projeto, no âmbito da Chamada Faps_Vale.

\section{REFERÊNCIAS}

1 MORAES, S.L. Contribuição ao entendimento do efeito de agente coloidisante no processo de pelotização de concentrado de minério de ferro. 2014. 138 p. Tese (Doutorado) - Escola Politécnica, Universidade São Paulo, São Paulo, 2014.

2 MOURÃO, J.M.; FREITAS, G.G.; GARIGLIO, E.; KLEIN, M.S. Influência da gênese dos minérios de ferro na etapa de formação de pelotas cruas no processo de pelotização. In: SIMPÓSIO BRASILEIRO DE MINÉRIO DE FERRO: Caracterização, Beneficiamento e Pelotização, 1., Ouro Preto, 1996. Anais. Ouro Preto: ABM, 1996. p. 75-94.

3 PENHA F.G.; SPIER, V.C.; DEBACHER N.A. Adsorção de xantatos sobre pirita. Química Nova, v. 24, n. 5, p. 612-615, 2001

4 SHAW, D.J. Introduction to colloid and surface chemistry. $4^{\text {th }}$. ed. Oxford: ButterworthHeinemann, 1992.

5 POTAPOVA, E. Adsorption of surfactants and polymers on iron oxides: implications for flotation and agglomeration of iron ore. 2011. 144f. Thesis (Doctor of Philosophy) Department of Civil, Environmental and Natural Resources Engineering, Division of Sustainable Process Engineering. Lulea University of Technology, Luleå, 2011. JONES, F.; FARROW, J.B.; BRONSWIJK, W. An infrared study of a polyacrylate flocculant adsorbed on hematite. Langmuir, v. 14, p. 6512-6517, 1998.

7 FARMER, V.C. (Ed.). The infrared spectra of minerals. London: Mineralogical Society, 1974. (Mineralogical Society Monograph, 4).

8 GOTIC, M.; MUSIC, S. Mossbauer, FT-IR and FE SEM investigation of iron oxides precipitated from FeSO4 solutions. Journal of Molecular Structure, v. 834-836, p. 445-453, May 2007.

9 HUANG, C.K.; KERR, P.F. Infrared study of the carbonate minerals. The American Mineralogist, v. 45, p. 311-324, 1960.

10 AHEMEN, I.; MELUDU, O.; ODOH, E. Effect of sodium carboxymethyl cellulose concentration on the photophysical properties of zinc sulfide nanoparticles. British Journal of Applied Science \& Technology, v. 3, n. 4, p.1228-1245, 2013.

11 MORAIS, E.C.; CORREA, G.G.; BRAMBILLA, R.; RADTKE, C.; BAIBICH, I.F.; SANTOS, J.H.Z. The interaction of encapsulated pharmaceutical drugs with a silica matrix. Colloids and Surfaces. B: Biointerfaces, v. 103, n. 1, p. 422-429, Mar. 2013. 